\title{
Métodos estatísticos para estudo de adaptabilidade e estabilidade fenotípica em soja
}

\author{
Waldir Camargos Júnior e Silva( ${ }^{(1)}$ e João Batista Duarte(1)
}

\begin{abstract}
(1)Universidade Federal de Goiás, Escola de Agronomia e Engenharia de Alimentos, Caixa Postal 131, CEP 74001-970 Goiânia, GO. E-mail: waldircamargo@bol.com.br, jbduarte@agro.ufg.br
\end{abstract}

\begin{abstract}
Resumo - O objetivo deste trabalho foi avaliar os métodos estatísticos de análise da interação de genótipos com ambientes (GxA), enfatizando a adaptabilidade e a estabilidade fenotípica. Utilizaram-se dados de produtividade de grãos de soja de sete experimentos em Goiás, testando 28 genótipos, dos quais quatro cultivares comerciais. Avaliaram-se os métodos Tradicional, Plaisted \& Peterson, Wricke, Finlay \& Wilkinson, Eberhart \& Russell, Verma, Chahal \& Murty, Toler, AMMI (additive main effect and multiplicative interaction), Hühn, Annicchiarico e Lin \& Binns. Avaliou-se a associação entre os métodos pela correlação de Spearman. Observou-se forte associação entre os de Plaisted \& Peterson e Wricke, cujo uso concomitante foi contra-indicado. A mesma conclusão é atribuída aos métodos Annicchiarico e Lin \& Binns, também fortemente associados, o que implica em classificações fenotípicas muito semelhantes. O uso de um deles, entretanto, é recomendado. Métodos baseados, exclusivamente, em coeficientes de regressão, devem ser utilizados em associação com outro, fundamentado na variância da interação GxA, ou em medidas estatísticas como a variância dos desvios da regressão. O uso combinado do método de Eberhart \& Russell e AMMI é outra indicação, em razão de suas correlações significativas com a maioria dos outros métodos e uma associação relativamente fraca entre eles.
\end{abstract}

Termos para indexação: Glycine max, interação GxA, adaptação produtiva, estabilidade de rendimento.

\section{Statistical methods to study phenotypic adaptability and stability in soybean}

\begin{abstract}
The objective of this work was to evaluate statistical methods of genotype by environment interaction (GE) analysis, emphasizing phenotypic adaptability and stability. Observed data of soybean grain yield were used, which were obtained from variety trials carried out in seven environments in Goiás State. Twenty eight genotypes were evaluated in each trial, and four of them were commercial varieties. The methods evaluated were Traditional, Plaisted \& Peterson, Wricke, Finlay \& Wilkinson, Eberhart \& Russell, Verma, Chahal \& Murty, Toler, AMMI (additive main effect and multiplicative interaction), Hühn, Annicchiarico and Lin \& Binns. In order to study the association among the methods, the correlation of Spearman was used. Plaisted \& Peterson and Wricke methods were always strongly associated, contraindicating their concomitant use. The Annicchiarico and Lin \& Binns methods also showed high correlation between themselves producing, therefore, very similar genotypic classifications. Thus, the simultaneous use of these procedures is not also recommended. The use of one of them, however, is counseled. In the case of methods based exclusively on regression coefficients, it should be considered their association to other method, based in the variance of the GE interaction, or the introduction of statistical measures as the variance of the regression deviations. The joint use of Eberhart \& Russell method and AMMI analysis is another recommendation, because their significant correlations with most of the other methods, and of a low correlation between them.
\end{abstract}

Index terms: Glycine max, GE interaction, yield adaptation, yield stability.

\section{Introdução}

Programas de melhoramento de plantas visam à obtenção de genótipos com alta produtividade, estabilidade de produção e ampla adaptabilidade aos mais variados ambientes da região para a qual são recomendados. A interação de genótipos com ambientes (GxA), definida como a resposta diferencial dos genótipos à variação do ambiente, dificulta a seleção de genótipos amplamente adaptados. Além disso, essa interação pode inflacionar as estimativas de variância genética, resultando em superestimativas dos ganhos genéticos, esperados com a seleção, e num menor êxito, dos programas de melhoramento (Duarte \& Vencovsky, 1999). 
Para diminuir o efeito da interação GxA, a condução dos experimentos no maior número possível de locais e anos é necessária, para se avaliar a magnitude da interação e seu possível impacto sobre a seleção e a recomendação de cultivares. A fim de tornar essa recomendação a mais segura possível, é necessário um estudo detalhado acerca da adaptabilidade e da estabilidade das cultivares, assim como de seus caracteres importantes economicamente. Vários métodos estatísticos têm sido propostos e utilizados em aplicações e, a cada dia, novos procedimentos vêm sendo apresentados com o objetivo de se interpretar melhor a interação GxA. Estudos dessa natureza são importantes para o melhoramento de plantas, uma vez que fornecem informações sobre o comportamento de cada genótipo ante as variações do ambiente.

As análises de adaptabilidade e estabilidade são, portanto, procedimentos estatísticos que permitem, de algum modo, identificar as cultivares de comportamento mais estável e que respondem previsivelmente às variações ambientais. Algumas dessas análises permitem, também, dividir os efeitos da interação GxA em efeitos de genótipos e de ambientes, revelando a contribuição relativa de cada um para a interação total (Rocha, 2002). Assim, a estimação dos parâmetros de adaptabilidade e estabilidade fenotípica tem sido uma forma muito difundida, entre os melhoristas de plantas, de avaliar novos genótipos antes de sua recomendação como cultivares.

O objetivo deste trabalho foi avaliar métodos estatísticos de análise da adaptabilidade e estabilidade fenotípica, em soja, aplicando-os a dados experimentais de produtividade de grãos, obtidos de ensaios conduzidos no Estado de Goiás, na safra 2002/2003.

\section{Material e Métodos}

Os dados foram obtidos em ensaios de avaliação de linhagens experimentais de soja, de ciclo precoce, conduzidos na safra 2002/2003, no Estado de Goiás. Os experimentos são coordenados pelo Convênio Cerrados, constituído pelo Centro Tecnológico para Pesquisas Agropecuárias (CTPA), pela Agência Rural do Estado de Goiás e pela Embrapa (Empresa Brasileira de Pesquisa Agropecuária). Foram avaliados 28 genótipos, dos quais quatro cultivares testemunhas (M-Soy 8001, Nina, Emgopa 316 e M-Soy 6101). Os ensaios foram conduzidos em Anápolis, Chapadão do Céu, Goiatuba, Mineiros, Montividiu e Senador Canedo. Em Senador Canedo foram conduzidos dois ensaios, denominados Senador Canedo I e Senador Canedo II, que corresponderam a diferentes épocas de plantio, 8/11/2002 e 25/11/2002, respectivamente.

Os experimentos foram instalados em delineamento em blocos completos casualizados, com quatro repetições. As parcelas foram formadas por quatro fileiras de plantas ( $5 \mathrm{~m}$ ), espaçadas em $0,5 \mathrm{~m}$ entre as fileiras. A área útil da parcela foi de $4 \mathrm{~m}^{2}$, tendo sido colhidas as duas fileiras centrais, desprezando-se $0,5 \mathrm{~m}$ de bordadura nas extremidades. Priorizou-se a análise dos dados de produtividade de grãos, expressos em $\mathrm{kg} \mathrm{ha}^{-1}$ e ajustados para $13 \%$ de umidade.

Inicialmente, foram realizadas análises individuais de variância, seguindo-se uma análise conjunta de variância. A fim de implementar tais análises, utilizou-se o procedimento GLM do aplicativo SAS (SAS Institute, 2002). $\mathrm{Na}$ análise conjunta avaliou-se, primeiramente, a homogeneidade das variâncias residuais dos experimentos $\left(\mathrm{QM}_{\mathrm{R}}\right)$ que, a princípio, foi verificada por meio do teste da razão entre o maior e o menor quadrado médio residual dos ensaios. Apesar da relativa homogeneidade observada - razão inferior a sete vezes, conforme Pimentel-Gomes (1990) -, até mesmo nos números de graus de liberdade dos resíduos (Tabela 1), implementouse também, para a análise da homogeneidade das $\mathrm{QM}_{\mathrm{R}}$, o teste de Bartlett (Ramalho et al., 2000). Isso, em

Tabela 1. Latitude, longitude, altitude, produtividade média de grãos $\left(\bar{Y}_{. j}\right)\left(\mathrm{kg} \mathrm{ha}^{-1}\right)$, variância residual $\left(\mathrm{QM}_{\mathrm{R}}\right)$ e graus de liberdade do resíduo $\left(\mathrm{GL}_{\mathrm{R}}\right)$, de ensaios de competição de linhagens de soja, de ciclo precoce de maturação, no Estado de Goiás, na safra 2002/2003.

\begin{tabular}{|c|c|c|c|c|c|c|}
\hline Ambiente & Altitude (m) & Latitude & Longitude & $\bar{Y}_{. j}$ & $\mathrm{QM}_{\mathrm{R}}$ & $\mathrm{GL}_{\mathrm{R}}$ \\
\hline Anápolis & 1.047 & $16^{\circ} 19^{\prime} 54^{\prime \prime} \mathrm{S}$ & $48^{\circ} 52^{\prime} 59^{\prime \prime} \mathrm{W}$ & $3.190,65$ & $87.799,56$ & 81 \\
\hline Chapadão do Céu & 810 & $18^{\circ} 25^{\prime} 48^{\prime \prime} \mathrm{S}$ & $52^{\circ} 53^{\prime} 24^{\prime \prime} \mathrm{W}$ & $2.068,14$ & $98.556,26$ & 81 \\
\hline Goiatuba & 810 & $17^{\circ} 59^{\prime} 20^{\prime \prime} \mathrm{S}$ & $49^{\circ} 21^{\prime} 58^{\prime \prime} \mathrm{W}$ & $4.012,82$ & $409.366,13$ & 81 \\
\hline Mineiros & 900 & $17^{\circ} 25^{\prime} 48^{\prime \prime} \mathrm{S}$ & $52^{\circ} 30^{\prime} 40^{\prime \prime} \mathrm{W}$ & $2.370,12$ & $58.829,20$ & 79 \\
\hline Montividiu & 900 & $17^{\circ} 28^{\prime} 12^{\prime \prime} \mathrm{S}$ & $51^{\circ} 16^{\prime} 48^{\prime \prime} \mathrm{W}$ & $3.179,22$ & $103.720,11$ & 80 \\
\hline Senador Canedo I & 720 & $16^{\circ} 43^{\prime} 19^{\prime \prime} \mathrm{S}$ & $49^{\circ} 07^{\prime} 26^{\prime \prime} \mathrm{W}$ & $3.069,17$ & $244.137,11$ & 81 \\
\hline Senador Canedo II & 720 & $16^{\circ} 43^{\prime} 19^{\prime \prime} \mathrm{S}$ & $49^{\circ} 07^{\prime} 26^{\prime \prime} \mathrm{W}$ & $2.990,88$ & $134.094,33$ & 81 \\
\hline
\end{tabular}


decorrência de certo relacionamento, verificado entre as médias dos experimentos e seus respectivos $\mathrm{QM}_{\mathrm{R}}$. Diante da heterocedasticidade detectada, procedeu-se, então, ao ajuste dos graus de liberdade do erro médio e da interação GxA, conforme o método de Cochran (1954), citado por Pimentel-Gomes (1990). Após esses ajustes foram feitas as interpretações relativas às significâncias do teste $\mathrm{F}$ (Snedecor) na análise da variância (ANOVA).

A avaliação da adaptabilidade e estabilidade fenotípica dos genótipos foi feita pelos seguintes métodos: Tradicional (citado por Oliveira, 1976), Plaisted \& Peterson (1959), Finlay \& Wilkinson (1963), Wricke (1965), Eberhart \& Russell (1966), Verma et al. (1978), AMMI (Zobel et al., 1988), Lin \& Binns (1988), Hühn (1990), Toler (1990) e Annicchiarico (1992). Tais análises foram realizadas com o uso dos programas computacionais, Genes (Cruz, 1997), Estabilidade (Universidade Federal de Lavras, 2000) e SAS (SAS Institute, 2002). As estatísticas adotadas por esses métodos são descritas a seguir.

A medida da estabilidade dos genótipos, pelo método Tradicional, consiste numa estimativa da variação de ambientes, dentro de cada genótipo. Seu estimador é:

$$
\mathrm{QM}_{(\mathrm{A} / \mathrm{Gi})}=\frac{\mathrm{r}}{(\mathrm{a}-1)}\left[\sum_{\mathrm{j}} \mathrm{Y}_{\mathrm{ij}}^{2}-\frac{\left(\mathrm{Y}_{\mathrm{i}}\right)^{2}}{\mathrm{a}}\right] \text {, }
$$

em que $\mathrm{Y}_{\mathrm{ij}}$ é a média do genótipo i (i=1, 2, ... g), no ambiente $\mathrm{j}(\mathrm{j}=1,2, \ldots$, a), e r é o número de repetições associado ao genótipo.

No método de Plaisted \& Peterson, o estimador do parâmetro que descreve a estabilidade é dado por:

$\hat{\theta}_{\mathrm{i}}=\frac{1}{\mathrm{~g}-1}\left[\sum_{\mathrm{i}^{\prime}=1}^{\mathrm{g}} \hat{\sigma}_{(\mathrm{ga})_{\text {ii' }}}^{2}\right]\left(\mathrm{i} \neq \mathrm{i}^{\prime}\right)$,

em que $\hat{\sigma}_{(\mathrm{ga}) \mathrm{ii}}^{2}$ é o componente da interação GxA, estimado por ANOVA, a partir da análise conjunta de todos os ambientes, e um par de genótipos que envolve o genótipo i.

A estatística de estabilidade do método de Wricke, denominada ecovalência $\left(\omega_{\mathrm{i}}\right)$, é estimada por: $\omega_{i}=r \sum_{j}\left(Y_{i j}-\bar{Y}_{i .}-\bar{Y}_{. j}+\bar{Y}_{. .}\right)^{2}$.

A estatística que descreve a estabilidade e a adaptabilidade de um genótipo, no método de Finlay \& Wilkinson, consiste no coeficiente de regressão linear simples da resposta individual do genótipo, transformada $\left(\mathrm{Y}_{\mathrm{ij}}^{\prime}\right)$, sobre a média ambiental $\left(\mathrm{X}_{\mathrm{j}}\right)$ : $\hat{\beta}_{i}^{\prime}=\frac{\sum_{j} Y_{i j}^{\prime} X_{j}-\left(\sum_{j} Y_{i j}^{\prime} \sum_{j} X_{j}\right) / a}{\sum_{j} X_{j}^{2}-\left(\sum_{j} X_{j}\right)^{2} / a}, \operatorname{com} Y_{i j}^{\prime}=\log \left(Y_{i j}\right) e$

$\mathrm{X}_{\mathrm{j}}=\frac{\sum_{\mathrm{i}} \mathrm{Y}_{\mathrm{ij}}^{\prime}}{\mathrm{g}}$.

Similarmente ao método anterior, mas com dados não transformados, o método de Eberhart \& Russell usa, na avaliação individual dos genótipos, a produtividade média do genótipo $\left(\mu_{\mathrm{i}}\right)$, o seu coeficiente de regressão $\left(\beta_{\mathrm{i}}\right)$ e a variância dos desvios dessa regressão $\left(\sigma_{\mathrm{di}}^{2}\right)$. Seus respectivos estimadores são dados por:

$\overline{\mathrm{Y}}_{\mathrm{i}}=\frac{\sum_{\mathrm{j}} \mathrm{Y}_{\mathrm{ij}}}{\mathrm{a}}$

$\hat{\beta}_{i}=\frac{\sum_{j} Y_{i j} I_{j}}{\sum_{j} I_{j}^{2}}$, em que $I_{j}=\frac{\sum_{j} Y_{i j}}{g}-\frac{\sum_{i} \sum_{j} Y_{i j}}{a g}$ (índice ambiental)

$\hat{\sigma}_{\mathrm{di}}^{2}=\frac{\left[\sum_{\mathrm{j}} \mathrm{Y}_{\mathrm{ij}}^{2}-\left(\sum_{\mathrm{j}} \mathrm{Y}_{\mathrm{ij}}\right)^{2} / \mathrm{a}\right]-\left(\sum_{\mathrm{j}} \mathrm{Y}_{\mathrm{ij}} \mathrm{I}_{\mathrm{j}}\right)^{2} / \sum_{\mathrm{j}} \mathrm{I}_{\mathrm{j}}^{2}}{\mathrm{a}-2}$

Com o mesmo princípio do método anterior, o método de Verma, Chahal \& Murty faz o ajuste de duas regressões lineares simples, separadamente, avaliando-se a resposta de cada genótipo a dois grupos de ambientes: o desfavorável, caracterizado por índices ambientais $\left(\mathrm{I}_{\mathrm{j}}\right)$ negativos, e o favorável, caracterizado por índices ambientais positivos. A fim de se estimar a regressão do segundo grupo de ambientes (favoráveis) e garantir continuidade às duas linhas de regressão, é incluído também nesse grupo o ambiente com o menor índice negativo.

O modelo matemático que descreve a resposta do genótipo i, no ambiente j, no método de Toler é um modelo não-linear dado por:

$Y_{i j}=\alpha_{i}+\left[Z_{j} \beta_{1 i}+\left(1-Z_{j}\right) \beta_{2 i}\right] \mu_{j}+\delta_{i j}+\bar{\varepsilon}_{i j}$,

em que $\mathrm{Y}_{\mathrm{ij}}$ é a média do genótipo i no ambiente j; $\alpha_{\mathrm{i}}$ é o parâmetro que reflete o valor da resposta do genótipo i, no ambiente médio $\left(\mu_{\mathrm{j}}=0\right.$, sendo $\mu_{\mathrm{j}}$ a variável independente); $\beta_{\mathrm{li}}$ e $\beta_{2 \mathrm{i}}$ são os coeficientes da regressão nãolinear, que medem a resposta do genótipo i às variações nos ambientes de qualidade inferior e superior, respectivamente; $Z_{\mathrm{j}}$ é uma variável marcadora, que assume os 
valores $Z_{j}=1$, se $\mu_{j} \leq 0$, e $Z_{j}=0$, se $\mu_{j}>0 ; \mu_{j}$ é o parâmetro que mede a qualidade ambiental; $\delta_{\mathrm{ij}}$ é o desvio dessa regressão; $\bar{\varepsilon}_{\mathrm{ij}}$ é o erro experimental médio.

Essa análise foi implementada com o auxílio do sistema computacional Estabilidade (Universidade Federal de Lavras, 2000).

O modelo descritivo da resposta média de um genótipo i, num ambiente j, na análise AMMI (Zobel et al., 1988), é: $\mathrm{Y}_{\mathrm{ij}}=\mu+\mathrm{g}_{\mathrm{i}}+\mathrm{a}_{\mathrm{j}}+\sum_{\mathrm{k}=1}^{\mathrm{n}} \lambda_{\mathrm{k}} \gamma_{\mathrm{ik}} \alpha_{\mathrm{jk}}+\rho_{\mathrm{ij}}+\varepsilon_{\mathrm{ij}}$.

Com base num modelo tradicional de análise conjunta (média geral - $\mu$; efeito genotípico - $g_{i}$; efeito de ambiente $-\mathrm{a}_{\mathrm{j}}$; e erro experimental médio $-\varepsilon_{\mathrm{ij}}$ ), o termo clássico da interação do genótipo i com o ambiente j, normalmente denotado por $(\mathrm{ga})_{\mathrm{ij}}$, é modelado como: $\sum_{\mathrm{k}=1}^{\mathrm{n}} \lambda_{\mathrm{k}} \gamma_{\mathrm{ik}} \alpha_{\mathrm{jk}}+\rho_{\mathrm{ij}}$, em que $\rho_{\mathrm{ij}}=\sum_{\mathrm{k}=\mathrm{n}+1}^{\mathrm{p}} \lambda_{\mathrm{k}}$. Os novos termos desse modelo resultam da chamada decomposição por valores singulares (DVS), da matriz de interações estimadas: $\mathrm{GA}_{(\mathrm{gxa})}=\left[(\hat{\mathrm{ga}})_{\mathrm{ij}}\right]$, com: $\left(\hat{g a g}_{\mathrm{ij}}=\mathrm{Y}_{\mathrm{ij}}-\overline{\mathrm{Y}}_{\mathrm{i} .}-\overline{\mathrm{Y}}_{\mathrm{j}}+\overline{\mathrm{Y}}_{\mathrm{.}}\right.$. A partir da DVS $\left(\mathrm{GA}=\sum \lambda_{\mathrm{k}} \gamma_{\mathrm{k}} \alpha_{\mathrm{k}}^{\prime}, \mathrm{k}=1,2, \ldots, \mathrm{p}\right.$, em que p é o posto da matriz ${ }^{k} \mathrm{~A}$ ), define-se: $\lambda_{\mathrm{k}}$ é o k-ésimo valor singular de GA (escalar); $\gamma_{\text {ik }}$ é o elemento correspondente ao i-ésimo genótipo, no vetor $\gamma_{\mathrm{k}}$ (vetor singular coluna); $\alpha_{\mathrm{jk}}$ é o elemento correspondente ao j-ésimo ambiente, no vetor $\alpha_{\mathrm{k}}^{\prime}$ (vetor singular linha).

O ajuste desse modelo foi implementado por meio de rotina computacional do SAS, desenvolvida e descrita por Duarte \& Vencovsky (1999). O método produz escores de componentes principais de interação para cada genótipo (IPCA $A_{i}$ ), que refletem a sua contribuição para a interação GxA. Assim, o genótipo com o(s) menor(es) escore(s), em valor absoluto, é o mais estável.

O método de Hühn utiliza-se de medidas estatísticas não-paramétricas da estabilidade $\left(S_{1}, S_{2}\right.$ e $\left.S_{3}\right)$, que são estimadas por:

$\mathrm{S}_{1 \mathrm{i}}=\frac{\sum_{\mathrm{j} j \mathrm{j}}\left|\mathrm{r}_{\mathrm{ij}}-\mathrm{r}_{\mathrm{ij}}\right|}{\mathrm{a}(\mathrm{a}-1) / 2} ; \mathrm{S}_{2 \mathrm{i}}=\frac{\sum_{\mathrm{j}}\left(\mathrm{r}_{\mathrm{ij}}-\overline{\mathrm{r}}_{\mathrm{i}}\right)^{2}}{\mathrm{a}-1} ; \mathrm{S}_{3 \mathrm{i}}=\frac{\sum_{\mathrm{j}}\left|\mathrm{r}_{\mathrm{ij}}-\overline{\mathrm{r}}_{\mathrm{i}}\right|}{\overline{\mathrm{r}}_{\mathrm{i}}}$, com $\overline{\mathrm{r}}_{\mathrm{i}}=\frac{\sum_{\mathrm{j}} \mathrm{r}_{\mathrm{ij}}}{\mathrm{a}}$, em que $\mathrm{S}_{1 \mathrm{i}}$ é a média das diferenças absolutas entre as classificações do genótipo i nos ambientes; $\mathrm{r}_{\mathrm{ij}}$ é a classificação do genótipo i, no ambiente $\mathrm{j} ; \mathrm{S}_{2 \mathrm{i}}$ é a variância das classificações do genótipo i nos ambientes; $\overline{\mathrm{r}}_{\mathrm{i}}$ éa média das classificações do genótipo i nos ambientes; e $S_{3 i}$ é a soma dos desvios absolutos de cada classificação, em relação à média das classificações. O genótipo com máxima estabilidade é aquele que apresentar valores iguais a zero, das três estatísticas $\left(\mathrm{S}_{1}, \mathrm{~S}_{2}\right.$ e $\left.\mathrm{S}_{3}\right)$.

$\mathrm{O}$ método de Annicchiarico baseia-se no chamado índice de confiança genotípico, estimado por: $\mathrm{I}_{\mathrm{i}(\mathrm{g})}=\hat{\mu}_{\mathrm{i}(\mathrm{g})}-\mathrm{z}_{(1-\alpha)}{\hat{z_{\mathrm{i}}(\mathrm{g})}}_{\mathrm{g}}$, considerando-se todos os ambientes, em que $\hat{\mu}_{\mathrm{i}(\mathrm{g})}$ é a média porcentual dos genótipos $\mathrm{i}$; $\hat{\sigma}_{\text {Zig) }}$ é o desvio-padrão dos valores $Z_{\mathrm{ij}}$, associado ao i-ésimo genótipo; $\mathrm{z}_{(1-\alpha)}$ é o percentil da função de distribuição normal padrão. O coeficiente de confiança adotado foi de $75 \%$, isto é, $\alpha=0,25$.

A estatística de estabilidade e adaptabilidade $\mathrm{P}_{\mathrm{i}}$, adotada pelo método de Lin \& Binns, é obtida por:

$\mathrm{P}_{\mathrm{i}}=\frac{\sum_{\mathrm{j}=1}^{\mathrm{n}}\left(\mathrm{Y}_{\mathrm{ij}}-\mathrm{M}_{\mathrm{j}}\right)^{2}}{2 \mathrm{a}}$,

em que $\mathrm{M}_{\mathrm{j}}$ é a produtividade máxima entre todos os genótipos, no j-ésimo ambiente. O genótipo estável é aquele que apresentar o menor índice $\mathrm{P}_{\mathrm{i}}$.

A associação entre os métodos foi avaliada pela correlação linear, aplicada às ordens de classificação genotípica (correlação de Spearman), obtidas em cada par de métodos. A ordem de estabilidade e adaptabilidade, de cada método, foi definida conforme o seu conceito de estabilidade e número de parâmetros.

Nos métodos Tradicional, Plaisted \& Peterson, Wricke e Lin \& Binns, que utilizam um só parâmetro de estabilidade, atribuiu-se a ordem de número 1 ao genótipo com a menor estimativa do respectivo parâmetro, e assim por diante, até a ordem de número g, atribuída ao genótipo com a maior dessas estimativas.

No método de Finlay \& Wilkinson, também foi designada uma ordem crescente, a partir do genótipo com a menor estimativa do parâmetro $\beta_{i}^{\prime}$. O mesmo procedimento foi adotado para o método AMMI, considerando-se a magnitude dos escores (valor absoluto) do primeiro componente principal de interação (IPCA $\left.{ }_{1}\right)$.

Para os métodos Eberhart \& Russell (com parâmetros $\beta_{\mathrm{i}}$ e $\left.\sigma_{\mathrm{d}}^{2}\right)$, Verma, Chahal \& Murty $\left(\beta_{1 \mathrm{i}}^{1} \mathrm{e} \beta_{2 \mathrm{i}}^{1}\right)$, Toler $\left(\beta_{\mathrm{li}} \mathrm{e}\right.$ $\left.\beta_{2 \mathrm{i}}\right)$ e Hühn $\left(S_{1}, S_{2}, S_{3}\right)$, inicialmente, atribuíram-se ordenações crescentes às estimativas genotípicas de cada um desses parâmetros. E, em seguida, para cada método e genótipo, calculou-se a média das ordens de seus respectivos parâmetros.

No método Annicchiarico, de modo inverso, atribuiu-se a ordem de número 1 ao genótipo com a maior estimativa do parâmetro $\mathrm{I}_{\mathrm{i}}$ (índice de confiança genotípico sob 
todos os ambientes); e assim por diante, até o g-ésimo genótipo, aquele com a menor dessas estimativas.

\section{Resultados e Discussão}

Os efeitos da interação GxA, basicamente representadas, neste caso, por uma interação do tipo genótipos $\mathrm{x}$ locais, apresentaram significância a 1\% de probabilidade (Tabela 2). Isso indica que o comportamento relativo dos genótipos foi influenciado distintamente pelas condições ambientais, marcados, sobretudo, pelas diferenças entre locais. Este fato dificulta a recomendação de cultivares para a região estudada (Cruz \& Castoldi, 1991; Vencovsky \& Barriga, 1992), pois não se pode, nessas circunstâncias, fazer uma recomendação uniforme para todos os locais, sem prejuízo considerável na produção obtida, relativamente à produção possível.

As estimativas de adaptabilidade e de estabilidade fenotípica dos genótipos, relativas a cada método, são apresentadas na Tabela 3. Quanto à associação entre os métodos, pôde-se verificar que cerca de $40 \%$ das correlações estimadas apresentaram significância estatística (Tabela 4). Isso sugere, como esperado, um grau de associação entre os métodos estudados, o que, contudo, não garante concordância geral entre eles. Observa-se que os métodos de Plaisted \& Peterson e Wricke mostraram uma correlação perfeita entre si e, conseqüentemente, correlações idênticas com os demais métodos, o que evidencia informações de mesma natureza, isto é, redundantes. Essa semelhança decorre do fato de que ambos usam a decomposição da soma de quadrados, da interação GxA, na derivação de seus parâmetros de estabilidade (Cruz \& Regazzi, 2001).

O método de Eberhart \& Russell apresentou altas correlações com os métodos em estudo, exceto com os métodos de Annicchiarico e de Lin \& Binns. Isso indica que o método é capaz de mensurar, razoavelmente, as informações de estabilidade e adaptabilidade dos outros métodos, podendo até substituí-los em determinadas circunstâncias. A ordenação dos genótipos pelo método de Eberhart \& Russell, como efetuada aqui, combina dois tipos de estabilidade: estabilidade biológica, pela ordem dos coeficientes de regressão, da menor para a maior estimativa, e estabilidade agronômica, pela ordem das estimativas $\hat{\sigma}_{\mathrm{d}}^{2}$. Esse fato pode explicar a associação geral do método com a maioria dos outros.

Os métodos de Annicchiarico e de Lin \& Binns apresentaram forte associação entre si $\left(r_{s}=0,96\right)$, o que também contra-indica seu uso concomitante (Tabela 4). Esse resultado concorda com o obtido por Atroch et al. (2000), em arroz de sequeiro. Os dois procedimentos captam informações diferentes dos demais métodos, o que é indicado pelas suas baixas correlações com a maioria deles. Avaliação semelhante foi implementada por Borges et al. (2000), em feijão, ao comparar os métodos de Toler (1990), Annicchiarico (1992) e Lin \& Binns (1988). Os autores defendem, também, uma complementaridade entre os três métodos e recomendam, assim, o seu uso conjunto. Em contrapartida, Farias et al. (1997), ao trabalhar com algodão, encontraram correlação significativa entre os métodos de Eberhart \& Russell e de Lin \& Binns. Os resultados desse estudo indicam que o uso de um desses métodos em combinação, por exemplo, com o de Eberhart \& Russell, pode fornecer informações adicionais e complementares sobre a estabilidade fenotípica em soja.

A análise AMMI também não se correlacionou com a maior parte dos métodos. A associação mais forte foi apresentada com os métodos de Plaisted \& Peterson e de Wricke, os quais possuem princípio estatístico semelhante, isto é, são baseados na contribuição genotípica para a interação GxA. A análise AMMI representa um avanço metodológico, pois permite descartar ruídos na interação GxA estimada por esses métodos. Assim, em razão desse aprimoramento, essa análise apresenta-se como um método mais apropriado a esses estudos.

Tabela 2. Análise conjunta de variância da produtividade de grãos ( $\mathrm{kg} \mathrm{ha}^{-1}$ ), de 28 genótipos de soja (ciclo precoce) testados em sete ambientes, no Estado de Goiás, na safra 2002/2003.

\begin{tabular}{lcrrrr}
\hline Fonte de variação & GL & Soma de quadrados & Quadrado médio & F & \multicolumn{1}{c}{ Pr>F } \\
\hline Blocos/ambientes & 21 & $7.786 .281,30$ & $370.775,30$ & 1,53 \\
Ambientes (A) & 6 & $264.059 .709,30$ & $44.009 .951,60$ & 181,15 \\
Genótipos (G) & 27 & $33.182 .829,70$ & $1.228 .993,70$ & 5,06 \\
Interação GxA & $114^{(1)}$ & $58.032 .458,00$ & $509.056,65$ & $<0,0001$ & 2,10 \\
Erro médio & $378^{(1)}$ & $91.835 .357,20$ & $242.950,68$ & - & $<0,0001$ \\
\hline
\end{tabular}

(1)Valores ajustados pelo método de Cochran (1954), citado por Pimentel-Gomes (1990), em razão de heterocedasticidade. 


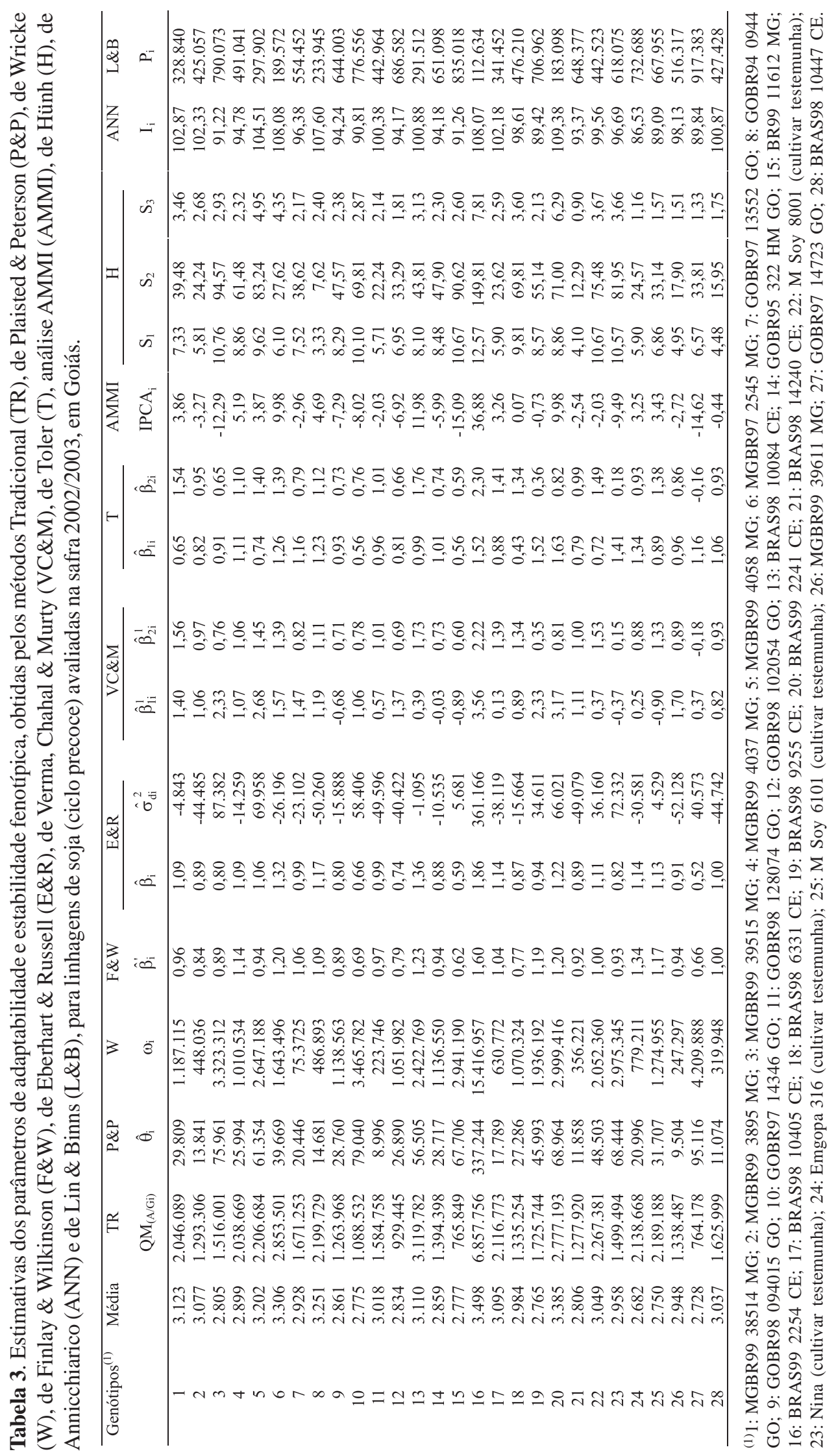


Tabela 4. Estimativas do coeficiente de correlação de Spearman, aplicado às ordens de adaptabilidade e estabilidade de cada par de métodos.

\begin{tabular}{|c|c|c|c|c|c|c|c|c|c|c|}
\hline Método $^{(1)}$ & $\mathrm{P} \& \mathrm{P}$ & $\mathrm{W}$ & $\mathrm{F} \& \mathrm{~W}$ & E\&R & VC\&M & $\mathrm{T}$ & AMMI & $\mathrm{H}$ & ANN & L\&B \\
\hline TR & 0,13 & 0,13 & $0,89 * *$ & $0,83 * *$ & $0,71 * *$ & $0,86^{* *}$ & 0,02 & 0,29 & $-0,56^{*}$ & $-0,72 * *$ \\
\hline $\mathrm{P} \& \mathrm{P}$ & & $1,00^{* *}$ & $-0,05$ & $0,69 * *$ & 0,19 & 0,26 & $0,72 * *$ & $0,79 * *$ & 0,08 & 0,13 \\
\hline W & & & $-0,05$ & $0,69^{* *}$ & 0,19 & 0,26 & $0,72 * *$ & $0,79 * *$ & 0,08 & 0,13 \\
\hline $\mathrm{F} \& \mathrm{~W}$ & & & & $0,73^{* *}$ & $0,58^{* *}$ & $0,88^{* *}$ & $-0,07$ & 0,05 & $-0,33$ & $-0,54 *$ \\
\hline$E \& R$ & & & & & $0,73 * *$ & $0,85 * *$ & $0,52 * *$ & $0,73 * *$ & 0,02 & $-0,06$ \\
\hline VC\&M & & & & & & $0,79 * *$ & 0,12 & 0,40 & $-0,31$ & $-0,36$ \\
\hline $\mathrm{T}$ & & & & & & & 0,30 & 0,36 & $-0,08$ & $-0,19$ \\
\hline AMMI & & & & & & & & 0,51 & $-0,06$ & 0,04 \\
\hline $\mathrm{H}$ & & & & & & & & & $-0,08$ & $-0,03$ \\
\hline ANN & & & & & & & & & & $0,96^{* * *}$ \\
\hline
\end{tabular}

(1)TR: Tradicional (citado por Oliveira, 1976); P\&P: Plaisted \& Peterson (1959); W: Wricke (1965); F\&W: Finlay \& Wilkinson (1963); E\&R: Eberhart \& Russell (1966); VC\&M: Verma et al. (1978); T: Toler (1990); AMMI: método AMMI; H: Hühn (1990); ANN: Annicchiarico (1992); L\&B: Lin \& Binns (1988). * e **Significativo a 5 e $1 \%$ de probabilidade, respectivamente.

Uma boa concordância foi verificada, ainda, entre os métodos que utilizam regressão, isto é, Finlay \& Wilkinson (1963), Eberhart \& Russell (1966), Verma et al. (1978) e Toler (1990). Esses métodos correlacionaram-se significativamente e mostraram as maiores correlações com os demais. Isso garante tranqüilidade aos usuários dos métodos de regressão, já difundidos em estudos dessa natureza; entretanto, indica, também, um certo nível de informação redundante entre eles. Ademais, a falta de associação desses métodos, exceto o de Eberhart \& Russell, com os que avaliam a contribuição genotípica para a interação GxA, reforça a tese de que alguma medida nesse sentido, como, por exemplo, a variância dos desvios da regressão ou os escores AMMI da interação GxA, deva ser utilizada em combinação com coeficientes de regressão.

Diante dessas constatações, observa-se, ainda, que o método de Eberhart \& Russell mostrou uma associação significativa com a análise AMMI, nesse caso, porém, de baixa magnitude $\left(r_{s}=0,52\right)$. Se esse fato vier a se confirmar em outras observações, pode-se inferir que esses métodos, de certa forma, se complementam, visto que o método de Eberhart \& Russell, em detrimento da análise AMMI, informa sobre a responsividade de cada genótipo ante a melhoria ambiental. Por sua vez, a análise AMMI produz uma estimativa da contribuição genotípica, para a interação GxA, livre de ruídos, que o método de Eberhart \& Russell não descarta. Essa correlação não considera, na análise de Eberhart \& Russell, o parâmetro média dos genótipos, embora este seja considerado na análise para fins de seleção. A inclusão das estimativas desse parâmetro, possivelmente, traria modificações como, por exemplo, aumento da correlação entre os métodos de Eberhart \& Russell e Lin \& Binns.

Finalmente, considerando-se a popularidade já alcançada por alguns métodos, alguns deles com vanta- gens como as destacadas anteriormente, os resultados deste estudo são indicativos de que o desenvolvimento horizontal de métodos de estabilidade (criação de novos métodos), em detrimento do desenvolvimento vertical, não seja uma prioridade científica atual.

\section{Conclusões}

1. Os métodos de Plaisted \& Peterson e Wricke apresentam grande concordância entre si, o que contra-indica seu uso concomitante.

2. Os métodos de Annicchiarico e Lin \& Binns mostram forte associação entre si e produzem classificações genotípicas similares quanto à estabilidade fenotípica; o uso simultâneo dos dois não é recomendado; entretanto, o uso de um deles em combinação com o de Eberhart \& Russell pode agregar informação à análise de estabilidade.

3. Métodos de estabilidade que se baseiam, exclusivamente, em coeficientes de regressão, não se associam àqueles baseados na contribuição genotípica para a interação GxA; é recomendável associá-los a um outro método com esse último princípio, ou introduzir medidas estatísticas dessa natureza.

4. A associação relativamente fraca $\left(r_{s}=0,52\right)$ entre os métodos de Eberhart \& Russel e AMMI, aliada à complementaridade de suas informações, permite indicar o seu uso combinado em estudos de estabilidade e adaptabilidade fenotípica.

\section{Referências}

ANNICCHIARICO, P. Cultivar adaptation and recommendation from alfafa trials in Northern Italy. Journal of Genetics and Breeding, v.46, p.269-278, 1992.

ATROCH, A.L.; SOARES, A.A.; RAMALHO, M.A.P. Adaptabilidade e estabilidade de linhagens de arroz de sequeiro 
testadas no Estado de Minas Gerais. Ciência e Agrotecnologia, v.24, p.541-548, 2000.

BORGES, L.C.; FERREIRA, D.F.; ABREU, A.F.B.; RAMALHO, M.A.P. Emprego de metodologias de avaliação da estabilidade fenotípica na cultura do feijoeiro (Phaseolus vulgaris L.). Revista Ceres, v.47, p.89-102, 2000.

CRUZ, C.D. Programa GENES: aplicativo computacional em genética e estatística (software). Viçosa: Imprensa Universitária, 1997. 442p.

CRUZ, C.D.; CASTOLDI, F.L. Decomposição da interação genótipos $\mathrm{x}$ ambientes em partes simples e complexa. Revista Ceres, v.38, p.422-430, 1991.

CRUZ, C.D.; REGAZZI, A.J. Modelos biométricos aplicados ao melhoramento genético. 2.ed. Viçosa: UFV, 2001. 390p.

DUARTE, J.B.; VENCOVSKY, R. Interação genótipos $\mathbf{x}$ ambientes: uma introdução à análise "AMMI”. Ribeirão Preto: Sociedade Brasileira de Genética, 1999. 60p. (Monografias, 9).

EBERHART, S.A.; RUSSELL, W.A. Stability parameters for comparing varieties. Crop Science, v.6, p.36-40, 1966.

FARIAS, F.J.C.; RAMALHO, M.A.P.R.; CARVALHO, L.P.; MOREIRA, J.A.N.; COSTA, J.N. Parâmetros de estabilidade propostos por Lin \& Binns (1988) comparados com o método da regressão. Pesquisa Agropecuária Brasileira, v.32, p.407-414, 1997.

FINLAY, K.W.; WILKINSON, G.N. The analysis of adaptation in a plant breeding programme. Australian Journal of Agricultural Research, v.14, p.742-754, 1963.

HÜHN, M. Nonparametric measures of phenotypic stability. Part I: theory. Euphytica, v.47, p.195-201, 1990.

LIN, C.S.; BINNS, M.R. A superiority measure of cultivar performance for cultivar x location data. Canadian Journal of Plant Science, v.68, p.193-198, 1988.
OLIVEIRA, A.C. Comparação de alguns métodos de determinação de estabilidade de plantas cultivadas. 1976. 64p. Dissertação (Mestrado) - Universidade de Brasília, Brasília.

PIMENTEL-GOMES, F. Curso de estatística experimental. 13.ed. Piracicaba: Nobel, 1990. 468p.

PLAISTED, R.L.; PETERSON, L.C. A technique for evaluating the ability of selections to yield consistently in different locations and seasons. American Potato Journal, v.36, p.381-385, 1959.

RAMALHO, M.A.P.; FERREIRA, D.F.; OLIVEIRA, A.C. A experimentação em genética e melhoramento de plantas. Lavras: Ufla, 2000. 326p.

ROCHA, M.M. Seleção de linhagens experimentais de soja para adaptabilidade e estabilidade fenotípica. 2002. 184p. Tese (Doutorado) - Escola Superior de Agricultura Luiz de Queiroz, Piracicaba.

SAS INSTITUTE (Cary, Estados Unidos). Software and services: system for Windows, versão 8.0: software. Cary, 2002.

TOLER, J.E. Patterns of genotypic performance over environmental arrays. 1990. 154p. Thesis (Ph.D.) - Clemson University, South Carolina, USA.

UNIVERSIDADE FEDERAL DE LAVRAS. Estabilidade (software): versão 3.0. Lavras: Ufla-DEX, 2000.

VENCOVSKY, R.; BARRIGA, P. Genética biométrica no fitomelhoramento. Ribeirão Preto: Sociedade Brasileira de Genética, 1992. 496p.

VERMA, M.M.; CHAHAL, G.S.; MURTY, B.R. Limitations of conventional regression analysis: a proposed modification. Theoretical and Applied Genetics, v.53, p.89-91, 1978.

WRICKE, G. Zur Berechnung der Ökovalenz bei Sommerweizen und Hafer. Pflanzenzuchtung, v.52, p.127-138, 1965.

ZOBEL, R.W.; WRIGHT, M.J.; GAUCH, H.G. Statistical analysis of a yield trial. Agronomy Journal, v.80, p.388-393, 1988.

Recebido em 29 de junho de 2004 e aprovado em 4 de abril de 2005 